\title{
On Some Metric Inequalities and Applications
}

\author{
Hassen Aydi $\mathbb{D}^{1,2,3}$ and Bessem Samet $\mathbb{D}^{4}$ \\ ${ }^{1}$ Nonlinear Analysis Research Group, Ton Duc Thang University, Ho Chi Minh City, Vietnam \\ ${ }^{2}$ Faculty of Mathematics and Statistics, Ton Duc Thang University, Ho Chi Minh City, Vietnam \\ ${ }^{3}$ China Medical University Hospital, China Medical University, Taichung 40402, Taiwan \\ ${ }^{4}$ Department of Mathematics, College of Science, King Saud University, P.O. Box 2455, Riyadh 11451, Saudi Arabia
}

Correspondence should be addressed to Hassen Aydi; hassen.aydi@tdtu.edu.vn

Received 4 August 2020; Revised 3 September 2020; Accepted 10 September 2020; Published 5 October 2020

Academic Editor: Huseyin Isik

Copyright @ 2020 Hassen Aydi and Bessem Samet. This is an open access article distributed under the Creative Commons Attribution License, which permits unrestricted use, distribution, and reproduction in any medium, provided the original work is properly cited.

We derive a new inequality in metric spaces and provide its geometric interpretation. Some applications of our result are given, including metric inequalities in Lebesgue spaces, matrices inequalities, multiplicative metric inequalities, and partial metric inequalities. Our main result is a generalization of that obtained by Dragomir and Gosa.

\section{Introduction}

In [1], Dragomir and Gosa established the following interesting inequality in metric spaces.

Theorem 1. (Dragomir-Gosa inequality).

Let $\mathscr{V}$ be a nonempty set equipped with a metric $\delta$. Let $N \geq 2$ be a natural number, $\left\{\xi_{i}\right\}_{i=1}^{N} \subset[0, \infty), \sum_{i=1}^{N} \xi_{i}=1$, and $\left\{\vartheta_{i}\right\}_{i=1}^{N} \subset \mathscr{V}$. Then,

$$
\sum_{i=1}^{N-1} \sum_{j=i+1}^{N} \xi_{i} \xi_{j} \delta\left(\vartheta_{i}, \vartheta_{j}\right) \leq \inf _{\vartheta \in \mathscr{V}} \sum_{i=1}^{N} \xi_{i} \delta\left(\vartheta_{i}, 9\right)
$$

Moreover, the inequality is optimal, in the sense that the multiplicative coefficient $C=1$ on the right-hand side of (1) (in front of inf) cannot be replaced by a smaller real number.

In the special case when $\xi_{i}=1 / N$, for all $i \in\{1,2, \cdots, N\}$, (1) reduces to

$$
\sum_{i=1}^{N-1} \sum_{j=i+1}^{N} \delta\left(\vartheta_{i}, \vartheta_{j}\right) \leq N \inf _{\vartheta \in \mathscr{V}} \sum_{i=1}^{N} \delta\left(\vartheta_{i}, \vartheta\right)
$$

Inequality (2) can be considered a polygonal-type inequality. Namely, it has the following geometric interpretation: let $\Omega$ be a polygon in a metric space with $N$ vertices and $\vartheta$ be an arbitrary point in the space. Then, the sum of all edges and diagonals of $\Omega$ is less than $N$-times the sum of the distances from $\vartheta$ to the vertices of $\Omega$.

In the same paper [1], the authors presented some interesting applications of inequality (1) to normed linear spaces and pre-Hilbert spaces.

In this paper, motivated by the above-mentioned work, a generalization of inequality (1) is obtained and its geometric interpretation is provided. Moreover, some applications of our result are given, including metric inequalities in Lebesgue spaces, matrices inequalities, multiplicative metric inequalities, and partial metric inequalities.

\section{Main Result and Some Consequences}

We first recall briefly the notion of metric spaces (see, e.g., [2]). Let $\mathscr{V}$ be a nonempty set and $\delta: \mathscr{V} \times \mathscr{V} \longrightarrow[0, \infty)$ be a given function. We say that $\delta$ is a metric on $\mathscr{V}$ if the following conditions hold: for all $\vartheta, \rho, \kappa \in \mathscr{V}$,

(i) $\delta(\vartheta, \rho)=0$, if and only if $\vartheta=\rho$

(ii) $\delta(\vartheta, \rho)=\delta(\rho, \vartheta)$ 
(iii) $\delta(\vartheta, \rho) \leq \delta(\vartheta, \kappa)+\delta(\kappa, \rho)$

In this case, we say that $(\mathscr{V}, \delta)$ is a metric space.

Let $\mathbb{N}$ be the set of positive natural numbers. Our main result is the following:

Theorem 2. Let $\mathscr{V}$ be a nonempty set equipped with a metric $\delta$. Let $m, N \in \mathbb{N}, \quad N \geq 2, \quad\left\{\xi_{i}\right\}_{i=1}^{N} \subset[0, \infty), \quad \sum_{i=1}^{N} \xi_{i}=1, \quad$ and $\left\{\vartheta_{i}\right\}_{i=1}^{N} \subset \mathscr{V}$. Then,

$$
\begin{aligned}
& \sum_{i=1}^{N-1} \sum_{j=i+1}^{N} \xi_{i} \xi_{j} \delta\left(\vartheta_{i}, \vartheta_{j}\right)^{m} \leq \frac{1}{2} \inf _{\vartheta \in \mathscr{V}}\left[2 \sum_{i=1}^{N} \xi_{i} \delta\left(\vartheta_{i}, \vartheta\right)^{m}\right. \\
& \left.+\sum_{k=1}^{m-1}\left(\begin{array}{l}
m \\
k
\end{array}\right)\left(\sum_{i=1}^{N} \xi_{i} \delta\left(\vartheta_{i}, \vartheta\right)^{k}\right)\left(\sum_{i=1}^{N} \xi_{i} \delta\left(\vartheta, \vartheta_{i}\right)^{m-k}\right)\right] .
\end{aligned}
$$

Moreover, the inequality is optimal, in the sense that the multiplicative coefficient $1 / 2$ on the right-hand side of (3) cannot be replaced by a smaller real number.

Proof. By the triangle inequality, for all $\vartheta \in \mathscr{V}$, one has

$$
\delta\left(\vartheta_{i}, \vartheta_{j}\right) \leq \delta\left(\vartheta_{i}, \vartheta\right)+\delta\left(\vartheta, \vartheta_{j}\right), \quad \mathrm{i}, j \in\{1,2, \cdots, N\},
$$

which yields

$$
\delta\left(\vartheta_{i}, \vartheta_{j}\right)^{m} \leq\left(\delta\left(\vartheta_{i}, \vartheta\right)+\delta\left(\vartheta, \vartheta_{j}\right)\right)^{m} .
$$

On the other hand, by the binomial theorem, one has

$$
\left(\delta\left(\vartheta_{i}, \vartheta\right)+\delta\left(\vartheta, \vartheta_{j}\right)\right)^{m}=\sum_{k=0}^{m}\left(\begin{array}{l}
m \\
k
\end{array}\right) \delta\left(\vartheta_{i}, \vartheta\right)^{k} \delta\left(\vartheta, \vartheta_{j}\right)^{m-k}
$$

where

$$
\left(\begin{array}{l}
m \\
k
\end{array}\right)=\frac{m !}{(m-k) ! k !} .
$$

Hence, combining (5) with (6), one obtains

$$
\delta\left(\vartheta_{i}, \vartheta_{j}\right)^{m} \leq \sum_{k=0}^{m}\left(\begin{array}{l}
m \\
k
\end{array}\right) \delta\left(\vartheta_{i}, \vartheta\right)^{k} \delta\left(\vartheta, \vartheta_{j}\right)^{m-k} .
$$

Multiplying the above inequality by $\xi_{i} \xi_{j}$ and taking the sum over $i$ and $j$, it holds that

$$
\sum_{i, j=1}^{N} \xi_{i} \xi_{j} \delta\left(\vartheta_{i}, \vartheta_{j}\right)^{m} \leq \sum_{i, j=1}^{N} \xi_{i} \xi_{j} \sum_{k=0}^{m}\left(\begin{array}{l}
m \\
k
\end{array}\right) \delta\left(\vartheta_{i}, \vartheta\right)^{k} \delta\left(\vartheta, \vartheta_{j}\right)^{m-k}
$$

Notice that due to the symmetry of $\delta$ and the fact that $\delta$ $(u, u)=0, u \in \mathscr{V}$, one has

$$
\sum_{i, j=1}^{N} \xi_{i} \xi_{j} \delta\left(\vartheta_{i}, \vartheta_{j}\right)^{m}=2 \sum_{i=1}^{N-1} \sum_{j=i+1}^{N} \xi_{i} \xi_{j} \delta\left(\vartheta_{i}, \vartheta_{j}\right)^{m}
$$

Furthermore, using that $\sum_{i=1}^{N} \xi_{i}=1$, one obtains

$$
\begin{aligned}
\sum_{i, j=1}^{N} \xi_{i} \xi_{j} \sum_{k=0}^{m}\left(\begin{array}{l}
m \\
k
\end{array}\right) \delta\left(\vartheta_{i}, \vartheta\right)^{k} \delta\left(\vartheta, \vartheta_{j}\right)^{m-k} \\
=\sum_{k=0}^{m}\left(\begin{array}{l}
m \\
k
\end{array}\right) \sum_{i, j=1}^{N} \xi_{i} \xi_{j} \delta\left(\vartheta_{i}, \vartheta\right)^{k} \delta\left(\vartheta, \vartheta_{j}\right)^{m-k} \\
=\sum_{k=0}^{m}\left(\begin{array}{l}
m \\
k
\end{array}\right)\left(\sum_{i=1}^{N} \xi_{i} \delta\left(\vartheta_{i}, \vartheta\right)^{k}\right)\left(\sum_{j=1}^{N} \xi_{j} \delta\left(\vartheta, \vartheta_{j}\right)^{m-k}\right) \\
=\sum_{k=0}^{m}\left(\begin{array}{l}
m \\
k
\end{array}\right)\left(\sum_{i=1}^{N} \xi_{i} \delta\left(\vartheta_{i}, \vartheta\right)^{k}\right)\left(\sum_{i=1}^{N} \xi_{i} \delta\left(\vartheta, \vartheta_{i}\right)^{m-k}\right) \\
=2 \sum_{i=1}^{N} \xi_{i} \delta\left(\vartheta_{i}, \vartheta\right)^{m}+\sum_{k=1}^{m-1}\left(\begin{array}{c}
m \\
k
\end{array}\right)\left(\sum_{i=1}^{N} \xi_{i} \delta\left(\vartheta_{i}, \vartheta\right)^{k}\right) \\
\quad \times\left(\sum_{i=1}^{N} \xi_{i} \delta\left(\vartheta, \vartheta_{i}\right)^{m-k}\right),
\end{aligned}
$$

i.e.,

$$
\begin{gathered}
\sum_{i, j=1}^{N} \xi_{i} \xi_{j} \sum_{k=0}^{m}\left(\begin{array}{l}
m \\
k
\end{array}\right) \delta\left(\vartheta_{i}, \vartheta\right)^{k} \delta\left(\vartheta, \vartheta_{j}\right)^{m-k}=2 \sum_{i=1}^{N} \xi_{i} \delta\left(\vartheta_{i}, \vartheta\right)^{m} \\
+\sum_{k=1}^{m-1}\left(\begin{array}{c}
m \\
k
\end{array}\right)\left(\sum_{i=1}^{N} \xi_{i} \delta\left(\vartheta_{i}, \vartheta\right)^{k}\right)\left(\sum_{i=1}^{N} \xi_{i} \delta\left(\vartheta, \vartheta_{i}\right)^{m-k}\right) .
\end{gathered}
$$

Hence, it follows from (9), (10), and (12) that

$$
\begin{aligned}
& \sum_{i=1}^{N-1} \sum_{j=i+1}^{N} \xi_{i} \xi_{j} \delta\left(\vartheta_{i}, \vartheta_{j}\right)^{m} \leq \frac{1}{2}\left[2 \sum_{i=1}^{N} \xi_{i} \delta\left(\vartheta_{i}, \vartheta\right)^{m}\right. \\
& \left.+\sum_{k=1}^{m-1}\left(\begin{array}{l}
m \\
k
\end{array}\right)\left(\sum_{i=1}^{N} \xi_{i} \delta\left(\vartheta_{i}, \vartheta\right)^{k}\right)\left(\sum_{i=1}^{N} \xi_{i} \delta\left(\vartheta, \vartheta_{i}\right)^{m-k}\right)\right] .
\end{aligned}
$$

Notice that the above inequality holds for all $\vartheta \in \mathscr{V}$. So, taking the infimum over $\vartheta \in \mathscr{V}$, (3) follows. 
Suppose now that there exists a certain constant $M>0$ such that

$$
\begin{aligned}
& \sum_{i=1}^{N-1} \sum_{j=i+1}^{N} \xi_{i} \xi_{j} \delta\left(\vartheta_{i}, \vartheta_{j}\right)^{m} \leq M\left[2 \sum_{i=1}^{N} \xi_{i} \delta\left(\vartheta_{i}, \vartheta\right)^{m}\right. \\
& \left.\quad+\sum_{k=1}^{m-1}\left(\begin{array}{l}
m \\
k
\end{array}\right)\left(\sum_{i=1}^{N} \xi_{i} \delta\left(\vartheta_{i}, \vartheta\right)^{k}\right)\left(\sum_{i=1}^{N} \xi_{i} \delta\left(\vartheta, \vartheta_{i}\right)^{m-k}\right)\right]
\end{aligned}
$$

Taking $N=2, \vartheta_{1} \neq \vartheta_{2}, \quad \xi_{1}=\lambda$, and $\xi_{2}=1-\lambda$, where $\lambda \in(0,1)$, one obtains

$$
\begin{aligned}
\lambda(1-\lambda) \delta\left(\vartheta_{1}, \vartheta_{2}\right)^{m} \leq & 2 M\left[\lambda \delta\left(\vartheta_{1}, \vartheta\right)^{m}+(1-\lambda) \delta\left(\vartheta_{2}, \vartheta\right)^{m}\right] \\
& +M \sum_{k=1}^{m-1}\left(\begin{array}{l}
m \\
k
\end{array}\right)\left[\lambda \delta\left(\vartheta_{1}, \vartheta\right)^{k}\right. \\
& \left.+(1-\lambda) \delta\left(\vartheta_{2}, \vartheta\right)^{k}\right]\left[\lambda \delta\left(\vartheta_{1}, \vartheta\right)^{m-k}\right. \\
& \left.+(1-\lambda) \delta\left(\vartheta_{2}, \vartheta\right)^{m-k}\right]
\end{aligned}
$$

for all $\vartheta \in \mathscr{V}$. In particular, for $\vartheta=\vartheta_{1}$, one deduces that

$$
\begin{aligned}
\lambda(1-\lambda) \delta\left(\vartheta_{1}, \vartheta_{2}\right)^{m} \leq & 2 M(1-\lambda) \delta\left(\vartheta_{1}, \vartheta_{2}\right)^{m} \\
& +M(1-\lambda)^{2} \delta\left(\vartheta_{1}, \vartheta_{2}\right)^{m} \sum_{k=1}^{m-1}\left(\begin{array}{l}
m \\
k
\end{array}\right),
\end{aligned}
$$

which yields (since $\vartheta_{1} \neq \vartheta_{2}$ )

$$
\lambda \leq 2 M+M(1-\lambda) \sum_{k=1}^{m-1}\left(\begin{array}{l}
m \\
k
\end{array}\right)
$$

Taking the limit as $\lambda \longrightarrow 1^{-}$in the above inequality, it holds that $M \geq 1 / 2$. The proof is then complete.

Remark 3. Taking $m=1$ in Theorem 2, (3) reduces to (1).

Corollary 4. Let $\mathscr{V}$ be a nonempty set equipped with a metric $\delta$. Let $m, N \in \mathbb{N}, N \geq 2$, and $\left\{\vartheta_{i}\right\}_{i=1}^{N} \subset \mathscr{V}$. Then,

$$
\begin{aligned}
\sum_{i=1}^{N-1} & \sum_{j=i+1}^{N} \delta\left(\vartheta_{i}, \vartheta_{j}\right)^{m} \\
\leq & \inf _{\vartheta \in \mathscr{V}}\left[N \sum_{i=1}^{N} \delta\left(\vartheta_{i}, \vartheta\right)^{m}+\frac{1}{2} \sum_{k=1}^{m-1}\left(\begin{array}{l}
m \\
k
\end{array}\right)\left(\sum_{i=1}^{N} \delta\left(\vartheta_{i}, \vartheta\right)^{k}\right)\right. \\
& \left.\times\left(\sum_{i=1}^{N} \delta\left(\vartheta, \vartheta_{i}\right)^{m-k}\right)\right] .
\end{aligned}
$$

Proof. Using (3) with

$$
\xi_{i}=\frac{1}{N}, \quad i \in\{1,2, \cdots, N\}
$$

(18) follows.

Remark 5. Taking $m=1$ in Corollary 4, (18) reduces to (2).

In the special case $m=2$, one deduces form Corollary 4 the following result.

Corollary 6. Let $\mathscr{V}$ be a nonempty set equipped with a metric $\delta$. Let $N \in \mathbb{N}, N \geq 2$, and $\left\{\vartheta_{i}\right\}_{i=1}^{N} \subset \mathscr{V}$. Then,

$$
\sum_{i=1}^{N-1} \sum_{j=i+1}^{N} \delta\left(\vartheta_{i}, \vartheta_{j}\right)^{2} \leq \inf _{\vartheta \in \mathscr{V}}\left[N \sum_{i=1}^{N} \delta\left(\vartheta_{i}, \vartheta\right)^{2}+\left(\sum_{i=1}^{N} \delta\left(\vartheta_{i}, \vartheta\right)\right)^{2}\right]
$$

Inequality (20) has the following geometric interpretation.

Corollary 7. Let $\Omega$ be a polygon in a metric space with $N$ vertices, and $\vartheta$ be an arbitrary point in the space. Then, the sum of the squares of all edges and diagonals of $\Omega$ is less than $N$-times the sum of the squares of the distances from $\vartheta$ to the vertices of $\Omega$ plus the square of the sum of the distances from $\vartheta$ to the vertices of $\Omega$.

Given $\rho>0$ and $\vartheta \in \mathscr{V}$, where $(\mathscr{V}, \delta)$ is a metric space, we denote by $B_{\vartheta} \overline{(}(\rho)$ the closed ball in $\mathscr{V}$ with center $\vartheta$ and radius $\rho$, namely,

$$
\left.B_{\vartheta} \overline{(} \rho\right)=\{u \in \mathscr{V}: \delta(\vartheta, u) \leq \rho\}
$$

Corollary 8. Let $\mathscr{V}$ be a nonempty set equipped with a metric $\delta$. Let $m, N \in \mathbb{N}, \quad N \geq 2, \quad\left\{\xi_{i}\right\}_{i=1}^{N} \subset[0, \infty), \quad \sum_{i=1}^{N} \xi_{i}=1$, and $\left\{\vartheta_{i}\right\}_{i=1}^{N} \subset \mathscr{V}$. Suppose that there exist $\rho>0$ and $\vartheta \in \mathscr{V}$ such that $\left\{\vartheta_{i}\right\}_{i=1}^{N} \subset B_{\vartheta}(\rho)$. Then,

$$
\sum_{i=1}^{N-1} \sum_{j=i+1}^{N} \xi_{i} \xi_{j} \delta\left(\vartheta_{i}, \vartheta_{j}\right)^{m} \leq 2^{m-1} \rho^{m}
$$

Proof. Since $\left\{\vartheta_{i}\right\}_{i=1}^{N} \subset B_{\vartheta} \overline{(}(\rho)$, one has

$$
\delta\left(\vartheta_{i}, \vartheta\right) \leq \rho, \quad i \in\{1,2, \cdots, N\}
$$

Hence, using (3), (23), and the fact that $\sum_{i=1}^{N} \xi_{i}=1$, one deduces that 


$$
\begin{aligned}
\sum_{i=1}^{N-1} & \sum_{j=i+1}^{N} \xi_{i} \xi_{j} \delta\left(\vartheta_{i}, \vartheta_{j}\right)^{m} \\
\leq & \frac{1}{2}\left[2 \sum_{i=1}^{N} \xi_{i} \delta\left(\vartheta_{i}, \vartheta\right)^{m}+\sum_{k=1}^{m-1}\left(\begin{array}{l}
m \\
k
\end{array}\right)\left(\sum_{i=1}^{N} \xi_{i} \delta\left(\vartheta_{i}, \vartheta\right)^{k}\right)\right. \\
& \left.\times\left(\sum_{i=1}^{N} \xi_{i} \delta\left(\vartheta, \vartheta_{i}\right)^{m-k}\right)\right] \leq \frac{1}{2}\left[2 \rho^{m}+\rho^{m} \sum_{k=1}^{m-1}\left(\begin{array}{l}
m \\
k
\end{array}\right)\right] \\
= & \frac{\rho^{m}}{2}\left[2+\sum_{k=0}^{m}\left(\begin{array}{c}
m \\
k
\end{array}\right)-2\right]=2^{m-1} \rho^{m} .
\end{aligned}
$$

The proof is complete.

Remark 9. Taking $m=1$ in Corollary 8 , one obtains Corollary 2 in [1].

\section{Applications}

3.1. A Metric Inequality in Lebesgue Spaces. Consider a measure space $(\chi, \mathscr{M}, \mu)$ and a real number $r \in[1, \infty)$. We denote by $L^{r}(\chi, \mathscr{M}, \mu)$ the space of measurable functions $f$ such that

$$
\int_{\chi}|f|^{r} d \mu<\infty
$$

Proposition 10. Let $m, N \in \mathbb{N}, N \geq 2,\left\{\xi_{i}\right\}_{i=1}^{N} \subset[0, \infty), \sum_{i=1}^{N}$ $\xi_{i}=1$, and $\left\{f_{i}\right\}_{i=1}^{N} \subset L^{r}(\chi, M, \mu)$. Then,

$$
\sum_{i=1}^{N-1} \sum_{j=i+1}^{N} \xi_{i} \xi_{j}\left(\int_{\chi}\left|f_{i}-f_{j}\right|^{r} d \mu\right)^{m / r} \leq \frac{1}{2} \inf _{f \in L^{r}(\chi, M, \mu)} \Lambda(f),
$$

where

$$
\begin{aligned}
\Lambda(f)= & 2 \sum_{i=1}^{N} \xi_{i}\left(\int_{\chi}\left|f_{i}-f\right|^{r} d \mu\right)^{m / r} \\
& +\sum_{k=1}^{m-1}\left(\begin{array}{c}
m \\
k
\end{array}\right)\left(\sum_{i=1}^{N} \xi_{i}\left(\int_{\chi}\left|f_{i}-f\right|^{r} d \mu\right)^{k / r}\right) \\
& \cdot\left(\sum_{i=1}^{N} \xi_{i}\left(\int_{\chi}\left|f_{i}-f\right|^{r} d \mu\right)^{(m-k) / r}\right),
\end{aligned}
$$

for all $f \in L^{r}(\chi, \mathscr{M}, \mu)$. Moreover, this inequality is optimal, in the sense that the multiplicative coefficient $1 / 2$ on the righthand side of (26) cannot be replaced by a smaller real number.

Proof. Consider the distance function

$$
\delta(f, g)=\left(\int_{\chi}|f-g|^{r} d \mu\right)^{1 / r}, \quad f, g \in L^{r}(\chi, \mathscr{M}, \mu) .
$$

Then, $\left(L^{r}(\chi, \mathscr{M}, \mu), \delta\right)$ is a metric space (see, e.g., [3]). Hence, using (3) with $\delta$ as defined above, (26) follows. The optimality of (26) follows from Theorem 2.

3.2. A Matrix Inequality. We denote by $\mathscr{M}_{n}(\mathbb{R})$ the set of square matrices of size $n \in \mathbb{N}, n \geq 2$, with real number coefficients. Let $M \in \mathscr{M}_{n}(\mathbb{R})$. We denote by $\rho(M)$ the spectral radius of $M$, namely,

$$
\rho(M)=\max \left\{\left|\lambda_{i}\right|: i=1,2, \cdots, n\right\},
$$

where $\lambda_{i}, i \in\{1,2, \cdots, n\}$, are the eigenvalues of $M$. We denote by $\sigma_{\max }(M)$ the largest singular value of $M$, namely,

$$
\sigma_{\max }(M)=\sqrt{\rho\left(M M^{t}\right)},
$$

where $M^{t}$ is the transpose of $M$. For more details on matrix analysis, see, for example, $[4,5]$.

Proposition 11. Let $m, N \in \mathbb{N}, N \geq 2,\left\{\xi_{i}\right\}_{i=1}^{N} \subset[0, \infty), \sum_{i=1}^{N}$ $\xi_{i}=1$, and $\left\{M_{i}\right\}_{i=1}^{N} \subset \mathscr{M}_{n}(\mathbb{R})$. Then,

$$
\sum_{i=1}^{N-1} \sum_{j=i+1}^{N} \xi_{i} \xi_{j}\left[\sigma_{\max }\left(M_{i}-M_{j}\right)\right]^{m} \leq \frac{1}{2} \inf _{M \in \mathscr{M}_{n}(\mathbb{R})} \mu(M),
$$

where

$$
\begin{aligned}
\mu(M)= & 2 \sum_{i=1}^{N} \xi_{i}\left[\sigma_{\max }\left(M_{i}-M\right)\right]^{m} \\
& +\sum_{k=1}^{m-1}\left(\begin{array}{c}
m \\
k
\end{array}\right)\left(\sum_{i=1}^{N} \xi_{i}\left[\sigma_{\max }\left(M_{i}-M\right)\right]^{k}\right) \\
& \cdot\left(\sum_{i=1}^{N} \xi_{i}\left[\sigma_{\max }\left(M-M_{i}\right)\right]^{m-k}\right) .
\end{aligned}
$$

Moreover, the inequality is optimal, in the sense that the multiplicative coefficient 1/2 on the right-hand side of (31) cannot be replaced by a smaller real number.

Proof. Consider the function $\delta: \mathscr{M}_{n}(\mathbb{R}) \times \mathscr{M}_{n}(\mathbb{R}) \longrightarrow[0, \infty)$ defined by

$$
\delta(A, B)=\sigma_{\max }(A-B), \quad A, B \in \mathscr{M}_{n}(\mathbb{R}) .
$$

Then, $\delta$ is a metric on $\mathscr{M}_{n}(\mathbb{R})$ (see, e.g., [5]). Hence, using (3) with $\delta$ as defined above, (31) follows. The optimality of (31) follows from Theorem 2.

3.3. A Multiplicative Metric Inequality. We first recall the notion of multiplicative metric spaces (see [6]). A multiplicative metric on a nonempty set $\mathscr{V}$ is a function $\sigma: \mathscr{V} \times \mathscr{V} \longrightarrow[1$, $\infty)$ satisfying the following properties: for all $\vartheta, \rho, \kappa \in \mathscr{V}$,

(i) $\sigma(\vartheta, \rho)=1$, if and only if $\vartheta=\rho$

(ii) $\sigma(\vartheta, \rho)=\sigma(\rho, \vartheta)$ 
(iii) $\sigma(\vartheta, \rho) \leq \sigma(\vartheta, \kappa) \sigma(\kappa, \rho)$

In this case, we say that $(\mathscr{V}, \sigma)$ is a multiplicative metric space.

Example 12. Let $\sigma:(0, \infty) \times(0, \infty) \longrightarrow[1, \infty)$ be the function defined by

$$
\sigma(\vartheta, \rho)= \begin{cases}\vartheta \rho^{-1}, & \text { if } \vartheta \geq \rho, \\ \rho \vartheta^{-1}, & \text { if } \vartheta<\rho .\end{cases}
$$

Then $\sigma$ is a multiplicative metric on $(0, \infty)$.

Proposition 13. Let $\mathscr{V}$ be a nonempty set equipped with a multiplicative metric $\sigma$. Let $m, N \in \mathbb{N}, N \geq 2,\left\{\xi_{i}\right\}_{i=1}^{N} \subset[0, \infty)$, $\sum_{i=1}^{N} \xi_{i}=1$, and $\left\{\vartheta_{i}\right\}_{i=1}^{N} \subset \mathscr{V}$. Then,

$$
\begin{aligned}
& \sum_{i=1}^{N-1} \sum_{j=i+1}^{N} \xi_{i} \xi_{j}\left[\ln \sigma\left(\vartheta_{i}, \vartheta_{j}\right)\right]^{m} \leq \frac{1}{2} \inf _{\vartheta \in \mathscr{V}}\left[2 \sum_{i=1}^{N} \xi_{i}\left[\ln \sigma\left(\vartheta_{i}, \vartheta\right)\right]^{m}\right. \\
& \left.+\sum_{k=1}^{m-1}\left(\begin{array}{l}
m \\
k
\end{array}\right)\left(\sum_{i=1}^{N} \xi_{i}\left[\ln \sigma\left(\vartheta_{i}, \vartheta\right)\right]^{k}\right)\left(\sum_{i=1}^{N} \xi_{i}\left[\ln \sigma\left(\vartheta, \vartheta_{i}\right)\right]^{m-k}\right)\right] .
\end{aligned}
$$

Moreover, the inequality is optimal, in the sense that the multiplicative coefficient 1/2 on the right-hand side of (35) cannot be replaced by a smaller real number.

Proof. Consider the function $\delta: \mathscr{V} \times \mathscr{V} \longrightarrow[0, \infty)$ defined by

$$
\delta(\vartheta, \rho)=\ln \sigma(\vartheta, \rho), \quad \vartheta, \rho \in \mathscr{V} .
$$

It can be easily seen that $\delta$ is a metric on $\mathscr{V}$. Then, using (3) with $\delta$ as defined above, (35) follows. The optimality of (35) follows from Theorem 2.

3.4. A Partial Metric Inequality. We first recall briefly some notions on partial metric spaces (see, e.g., [7-10]).

A partial metric on a nonempty set $\mathscr{V}$ is a function $\eta: \mathscr{V} \times \mathscr{V} \longrightarrow[0, \infty)$ satisfying the following properties: for all $\vartheta, \rho, \kappa \in \mathscr{V}$,

$$
\begin{aligned}
& \eta(\vartheta, \vartheta)=\eta(\rho, \rho)=\eta(\vartheta, \rho) \Leftrightarrow \vartheta=\rho, \\
& \eta(\vartheta, \vartheta) \leq \eta(\vartheta, \rho), \\
& \eta(\vartheta, \rho)=\eta(\rho, \vartheta), \\
& \eta(\vartheta, \rho) \leq \eta(\vartheta, \kappa)+\eta(\kappa, \rho)-\eta(\kappa, \kappa) .
\end{aligned}
$$

In this case, we say that $(\mathscr{V}, \eta)$ is a partial metric space.

Example 14. Let $\eta:[0, \infty) \times[0, \infty) \longrightarrow[0, \infty)$ be the function defined by

$$
\eta(\vartheta, \rho)=\max \{\vartheta, \rho\}, \quad \vartheta, \rho \geq 0 .
$$

Then, $\eta$ is a partial metric on $[0, \infty)$.
Proposition 15. Let $\mathscr{V}$ be a nonempty set equipped with a partial metric $\eta$. Let $m, N \in \mathbb{N}, N \geq 2,\left\{\xi_{i}\right\}_{i=1}^{N} \subset[0, \infty), \sum_{i=1}^{N}$ $\xi_{i}=1$, and $\left\{\vartheta_{i}\right\}_{i=1}^{N} \subset \mathscr{V}$. Then,

$\sum_{i=1}^{N-1} \sum_{j=i+1}^{N} \xi_{i} \xi_{j}\left[2 \eta\left(\vartheta_{i}, \vartheta_{j}\right)-\eta\left(\vartheta_{i}, \vartheta_{i}\right)-\eta\left(\vartheta_{j}, \vartheta_{j}\right)\right]^{m} \leq \frac{1}{2} \inf _{\vartheta \in \mathscr{V}} \Xi(\vartheta)$,

where

$$
\begin{aligned}
\Xi(\vartheta)= & 2 \sum_{i=1}^{N} \xi_{i}\left[2 \eta\left(\vartheta_{i}, \vartheta\right)-\eta\left(\vartheta_{i}, \vartheta_{i}\right)-\eta(\vartheta, \vartheta)\right]^{m} \\
& +\sum_{k=1}^{m-1}\left(\begin{array}{c}
m \\
k
\end{array}\right)\left(\sum_{i=1}^{N} \xi_{i}\left[2 \eta\left(\vartheta_{i}, \vartheta\right)-\eta\left(\vartheta_{i}, \vartheta_{i}\right)-\eta(\vartheta, \vartheta)\right]^{k}\right) \\
& \times\left(\sum_{i=1}^{N} \xi_{i}\left[2 \eta\left(\vartheta_{i}, \vartheta\right)-\eta\left(\vartheta_{i}, \vartheta_{i}\right)-\eta(\vartheta, \vartheta)\right]^{m-k}\right),
\end{aligned}
$$

for all $\vartheta \in \mathscr{V}$. Moreover, the inequality is optimal, in the sense that the multiplicative coefficient 1/2 on the right-hand side of (39) cannot be replaced by a smaller real number.

Proof. Consider the function $\delta: \mathscr{V} \times \mathscr{V} \longrightarrow[0, \infty)$ defined by

$$
\delta(\vartheta, \rho)=2 \eta(\vartheta, \rho)-\eta(\vartheta, \vartheta)-\eta(\rho, \rho), \quad \vartheta, \rho \in \mathscr{V} .
$$

It can be easily seen that $\delta$ is a metric on $\mathscr{V}$. Then, using (3) with $\delta$ as defined above, (39) follows. The optimality of (39) follows from Theorem 2.

\section{Conclusion}

A new inequality in metric spaces is proved. This inequality is a generalization of that derived by Dragomir and Gosa [1]. Moreover, we provided a geometric interpretation of our main result (see Corollary 7) and discussed some special cases including Lebesgue spaces, matrices inequalities, multiplicative metric inequalities, and partial metric inequalities.

\section{Data Availability}

The data used to support the study can be available upon request.

\section{Conflicts of Interest}

The authors declare that they have no competing interests regarding the publication of this paper.

\section{Authors' Contributions}

All authors contributed equally and significantly in writing this article. All authors read and approved the final manuscript. 


\section{Acknowledgments}

The second author is supported by Researchers' Supporting Project RSP-2020/4, King Saud University, Saudi Arabia, Riyadh.

\section{References}

[1] S. S. Dragomir and A. C. Gosa, "An inequality in metric spaces," Journal of The Indonesian Mathematical Society, vol. 11, no. 1, pp. 33-38, 2005.

[2] J. R. Giles, Introduction to the Analysis of Metric Spaces, Cambridge University Press, Cambridge, 1987.

[3] R. E. Castillo and H. Rafeiro, An Introductory Course in Lebesgue Spaces. CMS Books in Mathematics, Springer, Berlin, 2016.

[4] R. Bellman, Introduction to Matrix Analysis, McGraw-Hill, New York, NY, USA, 1974.

[5] R. Bhatia, Matrix Analysis, Springer, New York, NY, USA, 1997.

[6] A. E. Bashirov, E. M. Kurpınar, and A. Özyapıcı, "Multiplicative calculus and its applications," Journal of Mathematical Analysis and Applications, vol. 337, no. 1, pp. 36-48, 2008.

[7] L. Ćirić, B. Samet, H. Aydi, and C. Vetro, "Common fixed points of generalized contractions on partial metric spaces and an application," Applied Mathematics and Computation, vol. 218, no. 6, pp. 2398-2406, 2011.

[8] E. Karapinar, R. P. Agarwal, and H. Aydi, "Interpolative Reich-Rus-Ćirić type contractions on partial metric spaces," Mathematics, vol. 6, no. 11, p. 256, 2018.

[9] S. G. Matthews, "Partial metric topology," Annals of the New York Academy of Sciences, vol. 728, pp. 183-197, 1994.

[10] C. Vetro and F. Vetro, "Common fixed points of mappings satisfying implicit relations in partial metric spaces," Journal of Nonlinear Sciences and Applications, vol. 6, no. 3, pp. 152-161, 2013. 